\title{
A guiding oblique osteotomy cut to prevent bad split in sagittal split ramus osteotomy: a technical note
}

\author{
Gururaj Arakeri', Peter A. Brennan² \\ ${ }^{1}$ Department of Oral and Maxillofacial Surgery, Navodaya Dental College and Hospital, Raichur 584101, Karnataka, India. \\ ${ }^{2}$ Department of Oral and Maxillofacial Surgery, Queen Alexandra Hospital, Cosham, Portsmouth PO6 3LY, UK.
}

Address for correspondence: Dr. Gururaj Arakeri, Department of Oral and Maxillofacial Surgery, Navodaya Dental College and Hospital, Raichur 584101, Karnataka, India. E-mail: gururaj.arakeri@gmail.com

\begin{abstract}
Aim: To present a simple technical modification of a medial osteotomy cut which prevents its misdirection and overcomes various anatomical variations as well as technical problems. Methods: The medial osteotomy cut is modified in the posterior half at an angle of $15^{\circ}-20^{\circ}$ following novel landmarks. Results: The proposed cut exclusively directs the splitting forces downwards to create a favorable lingual fracture, preventing the possibility of an upwards split which would cause a coronoid or condylar fracture. Conclusion: This modification has proven to be successful to date without encountering the complications of a bad split or nerve damage.
\end{abstract}

Key words:

Guiding oblique osteotomy cut, lingual bad splits, medial cut, sagittal split ramus osteotomy

\section{INTRODUCTION}

Sagittal split osteotomy of the ramus may be the procedure which defined the evolution of the art and science of oral surgery. Although the basic design of the sagittal split ramus procedure evolved very quickly, the elimination of complications has taken longer. ${ }^{[1]}$ The procedure has been modified many times since its introduction by Trauner and Obwegeser. ${ }^{[2]}$ One modification frequently used is a shortened medial horizontal osteotomy, which, instead of extending the cut to the posterior border, is carried only to the lingual fossa posterior to the lingula. ${ }^{|3|}$ In the majority of cases, this technique allows for adequate splitting of the mandible. ${ }^{[3]}$ However, this modification is not devoid of complications, as the medial cut can be misdirected due to anatomic variability of the ramus, or an improperly directed osteotomy cut, resulting in a bad lingual split.

\begin{tabular}{|l|l|}
\hline \multicolumn{2}{|c|}{ Access this article online } \\
\hline Quick Response Code: & Website: \\
\hline & www.parjournal.net \\
\hline & \\
\hline
\end{tabular}

Various studies have reported an incidence of bad splits ranging from $1.7 \%$ to $9.1 \%{ }^{[4]}$ Although the most common unfavorable splits involve a buccal plate fracture, these bad lingual splits may result in serious complications including fracture of the lingual cortical plate, condylar neck and coronoid process. ${ }^{[4]}$

The purpose of this article is to suggest a modification of the medial osteotomy cut which will prevent misdirection while overcoming anatomical variations and technical problems.

\section{METHODS}

Surgical access for the sagittal split osteotomy is performed in the standard fashion. Following fine dissection over the anterior border of mandible, the insertion of the temporalis muscle is detached and elevated to the level of the sigmoid notch. The anterior ramus is then isolated with retraction of the soft tissues. The medial ramus is accessed by subperiosteal insertion of a malleable retractor above the foramen, and the inferior alveolar nerve is identified at the level of the lingula.

The medial osteotomy cut is directed paralle ${ }^{[3]}$ to the occlusal plane [Figure 1] at the level of superior aspect of lingula in the standard fashion, but ends at a point midway between the lingula and the ascending ramus. 


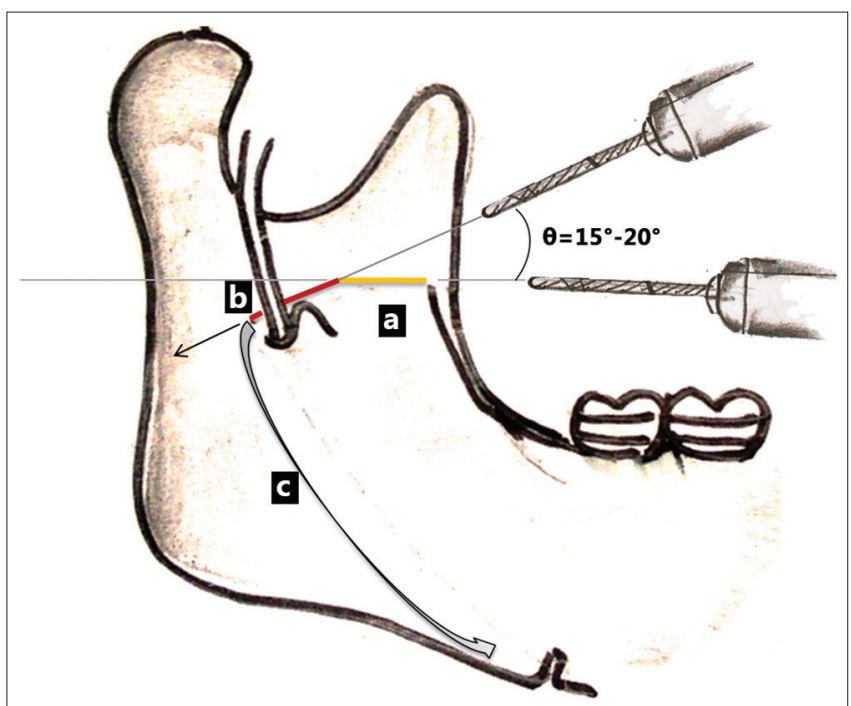

Figure 1: (a) Anterior half cut running parallel to occlusal plane (colored line); (b) posterior half cut (guiding oblique (GO) cut) with an angulation of " $\theta$ " running obliquely downwards and ending in lingual fossa; (c) favorable lingual split directed towards inferior body osteotomy by GO cut

This completes the anterior half of the traditional medial cut. The posterior half of the osteotomy cut is completed by directing the bur obliquely downwards at an angle of $15^{\circ}-20^{\circ}$ to the anterior half of the osteotomy cut which is parallel to occlusal plane. Care is taken to avoid damage to the inferior alveolar neurovascular bundle.

After completing the guiding oblique (GO) osteotomy cut, rest of the procedure is continued following routine standard technique. ${ }^{[3]}$

\section{RESULTS}

The GO cut extends downwards $3 \mathrm{~mm}$ posterior to the lingula from the point where the anterior half of the osteotomy cut was concluded. This creates an oblique angle between anterior and posterior osteotomy cuts and thus completes the modified medial ramus osteotomy. As the GO cut directs the splitting forces downwards, there is no possibility of the split propagating upwards to cause a coronoid or condylar fracture.

\section{DISCUSSION}

A major disadvantage of the traditional medial osteotomy cut is the effect that the anterior orientation of the bur has on the direction of the posterior cut. Another shortcoming of the cut is its abrupt termination at the posterior end. This produces a sharp angle at the junction of the buccal and lingual cortical plates of ramus of mandible. The forces applied in the sagittal split may concentrate at this angle, and the stress may be propagated in any direction to create bad splits. This is especially true in cases in which the posterior cut terminates in the monocortical area of the medial ramus rather in the bicortical region.

In contrast to the conventional medial cut, a simple modification which incorporates a GO cut in its posterior half minimizes the stress concentrated at this angle, and directs the cut downward to give a favorable lingual split. The surgical design also provides flexibility in placing the anterior half of the cut downwards in favorable bicortical region in cases of anatomical variation this avoids the possibility of coronoid fracture.

Advantages of a GO osteotomy cut in specific situations to prevent bad splits are as follows: (1) in regular situations a GO cut provides flexibility in adjusting the direction of the posterior medial cut if the anterior cut has been placed in an unfavorable direction; (2) in cases in which the ramal occlusal plane angle $e^{[3]}$ is $<70^{\circ}$, a parallel osteotomy would be directed superiorly, cutting the inferior portion of the neck of the condyloid process. ${ }^{[1]}$ To prevent this from occurring, it is advised to place the horizontal osteotomy cut at an altered angle of $10^{\circ}-15^{\circ}$ inferiorly. ${ }^{[3]}$ As the suggested modification already includes a $15^{\circ}$ bur angulation for the anterior cut, it is not necessary to adjust the osteotomy cut further; (3) in cases with a high lingula, there is an increased incidence of unfavorable fractures. ${ }^{[3]}$ A high lingula will place the medial osteotomy in a region of the mandibular ramus that has little or no cancellous bone. ${ }^{[5]}$ In this situation it is suggested that the medial osteotomy be angled from its typical location in the mid-ascending ramus up to the lingula of the medial ramus. ${ }^{[5]}$ If cancellous bone is not encountered, the medial cut is widened at the expense of cortex along the inferior aspect of the medial osteotomy until cancellous bone is seen. ${ }^{[5]}$ However, the widening creates an oblique medial cut directed upwards which increase the possibility of bad split. This complication is successfully avoided by the proposed modification which allows to place the anterior half of the cut in the favorable (bicortex) cancellous part of anterior ramus which is independent of the direction of posterior cut. A similar modification can be applied in cases of a thin mandible in which there is little cancellous bone; and (4) several investigators have demonstrated an increased risk of unfavorable fractures associated with the presence of third molars at the osteotomy site during sagittal split ramus osteotomy. ${ }^{[5-8]}$ The authors have observed a concentration of stress at the angle created by the buccal and lingual cortex of impacted third molar during the sagittal split which may result in a lingual plate fracture. For the same reason a lingual back cut ${ }^{[5]}$ posterior to the lingula is suggested. The addition of a lingual back cut helps direct the lingual fracture to a more favorable split at the inferior border of the osteotomy. ${ }^{[5]}$ The present GO cut acts as a back cut when directed 3-4 mm downwards with the same angulation which directs the lingual split laterally and inferiorly to the impacted molar providing a favorable split at the inferior border of the osteotomy.

In conclusion, splitting the straight medial cut into two components with angulation in the midpoint makes the anterior and posterior cuts independent of each other. Although there are many advantages and applications, a larger study is required to compare its versatility to that of the traditional medial cut. Nonetheless, the current modification has proven to be successful to date in the author's hands while avoiding the complications of a bad split or nerve damage. 


\section{REFERENCES}

I. Wyatt WM. Sagittal ramus split osteotomy: literature review and suggested modification of technique. Br J Oral Maxillofac Surg 1997;35:I 37-4I.

2. Trauner $\mathrm{R}$, Obwegeser $\mathrm{H}$. Operative oral surgery: the surgical correction of mandibular prognathism and retrognathia with consideration of genioplasty. Oral Surg Oral Med Oral Pathol 1957; 10:677-89.

3. Carleton AS, Schow SR, Peterson LJ. Prevention of the misdirected sagittal split. J Oral Maxillofac Surg 1986;44:8I-2.

4. Teltzrow T, Kramer FJ, Schulze A, Baethge C, Brachvogel P. Perioperative complications following sagittal split osteotomy of the mandible. J Craniomaxillofac Surg 2005;33:307-I3.

5. Cillo JE, Stella JP. Selection of sagittal split ramus osteotomy technique based on skeletal anatomy and planned distal segment movement: current therapy. J Oral Maxillofac Surg 2005;63:109-I4.

6. Mehra P, Castro V, Freitas RZ, Wolford LM. Complications of the mandibular sagittal split ramus osteotomy associated with the presence or absence of third molars. J Oral Maxillofac Surg 200 I;59:854-8.

7. Falter B, Schepers S, Vrielinck L, Lambrichts I, Thijs H, Politis C. Occurrence of bad splits during sagittal split osteotomy. Oral Surg Oral Med Oral Pathol Oral Radiol Endod 2010;1 10:430-5.

8. Doucet JC, Morrison AD, Davis BR, Robertson CG, Goodday R, Precious DS. Concomitant removal of mandibular third molars during sagittal split osteotomy minimizes neurosensory dysfunction. J Oral Maxillofac Surg 2012;70:2153-63.

How to cite this article: Arakeri G, Brennan PA. A guiding oblique osteotomy cut to prevent bad split in sagittal split ramus osteotomy: a technical note. Plast Aesthet Res 2015;2:127-9.

Source of Support: Nil, Conflict of Interest: None declared.

Received: 10-11-2014; Accepted: 30-03-2015 